\title{
The role of body size and fighting experience in predicting contest behaviour in the black field cricket, Teleogryllus commodus
}

\author{
Leeann T. Reaney • Jean M. Drayton • \\ Michael D. Jennions
}

Received: 21 January 2010 /Revised: 3 July 2010 / Accepted: 29 July 2010 /Published online: 10 August 2010

(C) Springer-Verlag 2010

\begin{abstract}
Body size strongly predicts fighting behaviour and outcome in many species, with the larger opponent usually winning contests. However, recent fighting experience can have a strong influence on the establishment of dominance hierarchies, with recent winners being more likely to win subsequent contests, while recent losers are more likely to lose. Recent fighting experience therefore has the potential to modify the effect of body size in determining contest behaviour and outcome. Here, we investigate whether recent fighting experience weakens the role of body size in predicting contest behaviour in the black field cricket, Teleogryllus commodus. We compared the role of body size in determining contest outcome during initial non-physical encounters and escalated aggressive physical encounters (grapples), as well as the probability of escalation occurring, in contests involving either a naïve or experienced smaller male against a naïve larger male. We found that recent fighting experience only affected contest outcome during non-physical encounters. Once a contest had escalated into grappling, the effect of previous wins and losses was no longer apparent and body size strongly predicted contest outcome. Thus, once males can directly assess their opponent's fighting ability, recent
\end{abstract}

Communicated by D. Gwynne

L. T. Reaney $(\bowtie)$

School of Biological and Chemical Sciences,

Queen Mary, University of London,

Mile End Road,

London E1 4NS, UK

e-mail: 1.reaney@qmul.ac.uk

L. T. Reaney $\cdot$ J. M. Drayton $\cdot$ M. D. Jennions

Evolution, Ecology and Genetics, Research School of Biology,

The Australian National University,

Canberra, Australian Capital Territory 0200, Australia fighting experience did not alter the effect of body size on contest behaviour and outcome.

Keywords Fighting behaviour . Contest outcome .

Body size $\cdot$ Recent fighting experience $\cdot$ Self-assessment .

Cricket - Teleogryllus commodus

\section{Introduction}

Males of many species engage in aggressive interactions when competing for valuable resources, such as shelter, food or mates. Fighting ability, or resource-holding potential (RHP), is often strongly correlated with body size and/ or weapon size, such that the larger of two competing males usually wins the contest (e.g. Pomfet and Knell 2006; Elias et al. 2008; Briffa 2008). However, an individual's fighting performance is often influenced by the outcomes of previous contests (Rutte et al. 2006). Recent wins generally increase the chances of an individual winning in the next encounter, while individuals that have experienced a recent loss are more likely to lose the next encounter (Hsu et al. 2006; Rutte et al. 2006). Fighting experience can therefore have a strong influence on the establishment of dominance hierarchies and alters contest outcome in many species (for review, see Hsu et al. 2006).

The effect of recent fighting experience on contest outcome is generally thought to result from changes in an individual's perception of its own fighting ability or social cues that identify past winners and losers to their opponents, such as physiological changes that occurred during earlier contests (Rutte et al. 2006; Hsu et al. 2009). If individuals re-assess their own fighting ability according to the outcome of previous encounters, fighting experience has the potential to override the effects of body size on 
contest outcome (Seebacher and Wilson 2007; Kasumovic et al. 2009). For example, in the crayfish, Procambarus clarkii, recent fighting experience altered the likelihood of winning subsequent contests independently of size disparities that would otherwise have accurately predicted outcomes (Daws et al. 2002). Similarly, in male copperheads, Agkistrodon contortrix, losing (but not winning) experience had a greater effect than body size on subsequent contest success and access to females (Schuett 1997). The decoupling of body size from fighting ability in determining contest outcome could occur if previous winners raise the estimate of their fighting ability after a fight, while previous losers lower their estimate. If true, winning a fight is likely to increase an individual's motivation in the next contest, while losing will result in a decrease in fighting motivation. An opponent would therefore have to invest more effort to win a fight against a previous winner but less effort to defeat a previous loser, compared to the effort required if RHP (often predicted based on body size) alone determined fighting success (i.e. in the absence of an asymmetry in perceived or actual resource value between opponents) (Whitehouse 1997; Humphries et al. 2006).

Recent fighting experience should not only affect the probability of winning a contest, but also the likelihood of initiating an aggressive encounter if experience alters perceived fighting ability. Once a contest escalates to physical interactions, however, opponents should be able to assess directly each other's fighting ability, and the importance of recent fighting experience should weaken. Recent fighting experience should therefore only strongly influence fighting behaviour during the early stages of a contest. In the hermaphroditic fish, Rivulus marmoratus, for example, a losing experience decreased the probability of an individual initiating a fight, while previous winners were more likely to attack and then escalate the fight (Hsu and Wolf 2001). Recent fighting experience, however, had no effect on the eventual outcome once a contest had escalated into an aggressive interaction (Hsu and Wolf 2001).

In this study, we investigate whether recent fighting experience weakens the role of body size in predicting contest behaviour and outcome in the black field cricket, Teleogryllus commodus (Orthoptera: Gryllinae). The advantage of field cricket contests is that both opponents use the same fighting behaviour and do not exhibit different roles, such as those found in hermit crabs (e.g. resident and intruder) (Briffa and Elwood 2001) and gammarids (Prenter et al. 2006). Each opponent therefore has the same potential sources of information that they can use during a contest, and differences in resource value are less likely. Any differences are therefore attributable to what each opponent has experienced prior to the contest. Male field crickets engage in highly structured, aggressive contests with rival males, with stereotypical fighting sequences. Aggressive encounters often begin with low-intensity activities, such as antennal contact and mandible flaring. Fights can then escalate into violent wrestling and biting. Once defeated, the loser typically avoids any further physical contact, while the winner often performs an acoustic display. This makes the outcome of contests readily determined (see Hofman and Stevenson 2000 for a detailed description). Fighting ability is strongly correlated with body size in T. commodus and other field crickets (Savage et al. 2004; Shackleton et al. 2005) and previous studies show that contests are often resolved by assessing asymmetries in size (Hack 1997; Hofman and Schildberger 2001; Briffa 2008).

Many studies focus on how a difference in RHP affects contest dynamics, but recent studies have shown that, sometimes, only the RHP of the weaker opponent is important (mutual versus self assessment) (Taylor and Elwood 2003; Arnott and Elwood 2009). We therefore investigated the individual contribution of each opponent's body size (a standard measure of RHP) in determining contest behaviour and outcome in T. commodus. We tested how contest behaviour and outcome were influenced by the absolute size of the smaller and larger opponents, respectively, in contests between either two naïve males or a pair of males, where the smaller male had previously encountered rival males. If recent fighting experience alters a male's perception of his fighting ability, then experienced males are expected to perceive cues associated with the costs and benefits of the contest differently to naïve males during the early stages of the contest (Hsu et al. 2006). We therefore predicted that:

(a) Body size asymmetry will more accurately predict the outcome of initial, non-escalated encounters, when both males are naïve rather than when one of them has had previous fighting experience. Specifically, small males with recent winning experience should both escalate and win more non-physical encounters than naïve small males, who, in turn, should escalate and win more often than small males with recent losing experience.

(b) Once a contest escalates into an aggressive physical encounter, however, recent fighting experience will have little or no effect on the role of body size asymmetry or the absolute size of the smaller male in predicting the outcome of contests because fighting ability is assessed directly by opponents.

\section{Methods}

Study animal and rearing conditions

Gravid female T. commodus were collected from the Smith's Lake area in New South Wales, Australia, in March 
2006 and placed in a temperature-controlled room $\left(26-28^{\circ} \mathrm{C}\right)$ with a 12:12-h light-dark cycle. Females were housed individually and provided with a Petri dish of moist cotton wool for oviposition. The newly hatched $\mathrm{F}_{1}$ generation were housed in communal tubs $(11 \times 7 \times 17 \mathrm{~cm})$ according to family $(n=75)$ and provided with high-protein fish food pellets and water ad libitum. Males do not appear to engage in agonistic interactions in the communal tanks as early-stage nymphs (personal observations). When nymphs were approximately 3 weeks old, we transferred them into individual plastic containers $(8 \times 8 \times 5 \mathrm{~cm})$ with the same food and water regime. We then checked daily for eclosion into adulthood, which occurs after another 32-168 days in isolation. Once they reached adulthood, males were fed cat food (KitKat Krunch) and water ad libitum until tested.

\section{Experimental design}

When adult males were 10-15 days old (days posteclosion), test males were weighed on a Sartorius balance $( \pm 0.1 \mathrm{mg})$ and marked with enamel paint on their pronotum for identification. Male body mass was used as a measure of individual RHP. Body mass is strongly positively correlated to male pronotum size in $T$. commodus (unpublished data) and has previously been used as a measure of fighting ability (Shackleton et al. 2005). Two test males of similar age were then placed in a plastic container $(14 \times 13 \times 20 \mathrm{~cm})$ with a Sony digital video camera placed overhead to record the staged contest (see below). In all contests, males were from different families.

Males were paired so that the size difference between them was moderate (if the difference is too great, no fights will ensue as the smaller male will simply flee). This resulted in a moderate correlation in size between rival males $(r=0.676$, $n=239, p<0.001)$. The mean size difference in the 'naïve' and the 'experienced' treatments did not differ significantly $\left(t_{237}=0.58, p=0.561\right.$ : naïve: $161.37 \pm 78.34 \mathrm{mg}$; experienced: $155.31 \pm 77.56 \mathrm{mg}$; mean $\pm \mathrm{SD}$ ).

In the 'naïve' treatment contests, both test males were naïve with no previous fighting experience. We staged 157 contests between two naïve males. Pairs that failed to interact within 10 min were excluded from analysis $(n=8)$.

In the 'experienced' treatment, the smaller of the two test males was first allowed to acquire contest experience before conducting the final staged contest. To allow the smaller of the two test males to gain fighting experience, he was first placed on his own in an $8 \times 8 \times 5 \mathrm{~cm}$ container. We then sequentially introduced six stock opponents to allow the test male to gain fighting experience. Stock opponents were 15-20 days old and all had previous fighting experience. To encourage equal winning and losing experience, the size of the stock male relative to the test male was alternated between contests. Each test male therefore encountered an equal proportion of larger and smaller opponents. If the pair did not interact after $5 \mathrm{~min}$, they were separated and the next stock male was introduced. Each test male was introduced to only six stock males. Test males were allowed to rest for 5 min between contests. We recorded whether the test male won, lost or did not interact with each stock male opponent. Individual fighting experience was classified using two measures: last fight experience (win/loss) and the proportion of wins (number of wins divided by the total number of introductions where the two males interacted). Overall, $54 \%$ of test males lost more that half their contests, while $76 \%$ lost their last fight.

Winner effects deteriorate rapidly in some species (e.g. $3 \mathrm{~h}$ in sticklebacks, Bakker et al. 1989; $1 \mathrm{~h}$ in pumpkinseed fish, Chase et al. 1994, but see Hsu and Wolf 1999 and Seebacher and Wilson 2007 for long lasting winner effects) and flight behaviour has been shown to restore aggression by losers in crickets (Hofman and Stevenson 2000). Experienced males were therefore only allowed a rest for a period of $1 \mathrm{~h}$ in a small container, with access to food and water, before we staged and recorded the experimental contest with a larger, naïve opponent. This allowed the smaller male to recover from the previous contests but, presumably, was a short enough interval so that they could remember their recent fighting experience (see Results). We staged 92 contests where an experienced, smaller male encountered a larger, naïve opponent. Two pairs failed to interact within $10 \mathrm{~min}$ and were therefore excluded from subsequent analysis.

\section{Behavioural data}

We noted the degree of escalation and the outcome of each staged contest. Initially, males will make antennae contact, after which one male either retreats and avoids further contact or is aggressively pursued by the other 'winning' male (non-physical encounters). Alternatively, if, after the initial antennae contact, both males decide to fight, an aggressive physical encounter ensues (referred to as 'grappling' from here onwards). A grapple was defined as an interaction in which the two males interlocked mandibles and wrestled until one produced an aggressive call (the winner) and the other continually retreated and avoided further contact (the loser). In general, stable dominance relationships are formed between two males and their relative status is established in the first encounter (Shackleton et al. 2005).

We measured the duration of grapples as the start and end of these encounters is easily determined. This is not the case for non-physical encounters. Furthermore, grapples represent the most costly phase of a contest, when males can directly assess their opponent's RHP. Grapple duration 
was measured from when the males first made physical contact until a clear winner was determined. If males separated while grappling and stopped interacting without a clear winner, we halted timing and continued timing once the pair reinitiated the grapple. We measured grapple duration as the interval between the first and last contact of the first aggressive interaction that led to a winner.

\section{Statistical analysis}

In general, our analysis approach was a two-stage process. First, we tested whether there was a difference between the naïve and experienced treatment for the response variable of interest, and we looked at the effect of predictor variables within each treatment. Interpretation is therefore based on the effect of gaining experience (irrespective of whether this was of winning or losing). Second, within the experienced treatment trials, we tested for differences between losers and winners (based on 'last fight' or 'proportion of fights won'). This approach meant that tests were orthogonal and did not require testing for multiple pair-wise comparisons between naïve, experienced winner and experienced loser contests. To test whether the difference between naïve and experienced treatment trials was driven by experienced winners or experienced losers, or whether it was independent of winner-loser status, we compared the outcome of contests involving a smaller naïve male to those where the smaller male had either recent losing experience or recent winning experience using separate G-tests on a $2 \times 2$ contingency table.

We tested whether larger and smaller opponent size predicted fighting behaviour and contest outcome, rather than using the size difference or relative size of two opponents (RHP difference). This allowed us to examine the individual contribution of each male's size to determining fighting behaviour at each stage of a contest (Arnott and Elwood 2009). We used adjusted (type III) sum of squares to control for the size of the opponent.

We determined if recent fighting experience changed how male size is related to the outcome of non-physical and physical encounters (grapples) (i.e. who won) and whether or not the contest escalated to grappling by comparing coefficients from logistical regressions (predictors: small and large male size) for contests when the smaller opponent was either naïve or experienced. We calculated separate effect sizes $(r)$ for the influence of small and large opponent size in each regression and tested whether they differed between the two types of staged contests (Zar 1999). This is equivalent to testing for a size by fight type interaction, but has the advantage that the relative magnitude of the effect in each fight type is more clearly identifiable. We then performed separate linear regressions to determine how three variables (winner size, loser size and size difference) predicted grapple duration (log-transformed to approximate normality) for each contest type (i.e. naïve or experienced smaller male) (following methods in Taylor and Elwood 2003). We again compared the effect sizes for each predictor variable between the two types of staged contests.

Finally, looking only at staged contests involving an experienced smaller male, we performed separate G-tests on a $2 \times 2$ contingency table to determine whether last fight experience affected (a) contest outcome and (b) whether the contest escalated. We then ran separate logistic regressions to determine how the proportion of fights won while gaining experience affected contest outcome and escalation. We used a $t$ test to compare grapple duration between males that won and lost their last fight, and ran a linear regression to test whether the proportion of fights won predicted grapple duration. We used SPSS version 17 (SPSS Inc., Chicago, IL, USA) for all analyses.

\section{Results}

Does previous experience alter the role of body size on fight behaviour?

\section{Outcome of non-physical encounters}

Non-physical encounters (chases and avoidances) between two naïve males were won by the larger opponent in $52 \%$ of fights (binomial test: $p=0.88, n=42$ ), while contents involving a smaller, experienced male were won by the larger opponent in $85 \%$ of cases (binomial test: $p<0.001$; $n=46$ ). Non-physical encounters involving a smaller, experienced male were therefore significantly more likely to end with the larger opponent winning $(G=11.15, \mathrm{df}=1$, $p<0.001)$. There was no significant difference between the outcome of non-physical encounters with a smaller opponent, when he was naïve or had a recent winning experience $(G=0.22, \mathrm{df}=1, p=0.64)$. However, smaller opponents with recent losing experience were significantly less likely to win these encounters compared to naïve opponents $(G=17.75, \mathrm{df}=1, p<0.001)$. Thus, the main effect of experience was driven by males with a recent losing experience. There was no significant effect of smaller or larger opponent size on contest outcome for either type of staged contest (Table 1, letter A).

\section{Contest escalation}

In total, $72 \%$ of contests between two naïve males $(n=$ 149) escalated into a grapple where males made physical contact with each other, compared to $49 \%$ of contests that involved a smaller, experienced male $(n=90)(G=12.57$, $\mathrm{df}=1, p<0.001)$. The number of contests that escalated 
Table 1 The effect of larger and smaller opponent size on (A) outcome of non-physical encounters, (B) the probability of escalating and (C) the outcome of grapples in contests involving either a naïve or experienced smaller male against a larger naïve opponent

\begin{tabular}{|c|c|c|c|c|c|c|}
\hline Predictor variables & $b(\mathrm{SE})(\times 1,000)$ & Wald $\chi^{2}$ & $p$ & Effect size $(r)$ & $Z$ value & $p$ (effect size difference) \\
\hline \multicolumn{7}{|l|}{ A: Non-physical encounters } \\
\hline \multicolumn{7}{|l|}{ Larger opponent size } \\
\hline Naïve smaller opponent $(n=42)$ & $1.412(5.222)$ & 0.073 & 0.787 & 0.042 & \multirow[t]{2}{*}{0.059} & \multirow[t]{2}{*}{0.953} \\
\hline Experienced smaller opponent $(n=46)$ & $1.106(5.678)$ & 0.038 & 0.846 & 0.029 & & \\
\hline \multicolumn{7}{|l|}{ Smaller opponent size } \\
\hline Naïve smaller opponent $(n=42)$ & $2.765(3.948)$ & 0.490 & 0.484 & 0.11 & \multirow[t]{2}{*}{0.298} & \multirow[t]{2}{*}{0.766} \\
\hline Experienced smaller opponent $(n=46)$ & $3.028(6.149)$ & 0.242 & 0.622 & 0.173 & & \\
\hline \multicolumn{7}{|l|}{ B: Contest escalation } \\
\hline \multicolumn{7}{|l|}{ Larger opponent size } \\
\hline Naïve smaller opponent $(n=149)$ & $-0.521(2.763)$ & 0.036 & 0.85 & 0.016 & \multirow[t]{2}{*}{0.533} & \multirow[t]{2}{*}{0.594} \\
\hline Experienced smaller opponent $(n=90)$ & $-2.518(3.037)$ & 0.687 & 0.407 & 0.087 & & \\
\hline \multicolumn{7}{|l|}{ Smaller opponent size } \\
\hline Naïve smaller opponent $(n=149)$ & $5.546(2.616)$ & 4.494 & 0.034 & 0.174 & \multirow[t]{2}{*}{0.857} & \multirow[t]{2}{*}{0.392} \\
\hline Experienced smaller opponent $(n=90)$ & $1.717(3.051)$ & 0.317 & 0.573 & 0.059 & & \\
\hline \multicolumn{7}{|l|}{ C: Grapples } \\
\hline \multicolumn{7}{|l|}{ Larger opponent size } \\
\hline Naïve smaller opponent $(n=107)$ & $-14.818(4.587)$ & 10.433 & 0.001 & 0.312 & \multirow[t]{2}{*}{1.577} & \multirow[t]{2}{*}{0.115} \\
\hline Experience smaller opponent $(n=44)$ & $1.136(5.313)$ & 0.046 & 0.831 & 0.032 & & \\
\hline \multicolumn{7}{|l|}{ Smaller opponent size } \\
\hline Naïve smaller opponent $(n=107)$ & $12.005(4.016)$ & 8.936 & 0.003 & 0.289 & \multirow[t]{2}{*}{0.390} & \multirow[t]{2}{*}{0.696} \\
\hline Experience smaller opponent $(n=44)$ & $7.903(5.223)$ & 2.290 & 0.130 & 0.228 & & \\
\hline
\end{tabular}

The $Z$ values and $p$ values in the last two columns are a test for a difference in the effect size between the two types of staged contests (naive vs experienced smaller male). Significant $p$ values are in italics

did not differ between contents involving a smaller male that was naïve or had a recent winning experience $(G=0.12, \mathrm{df}=1, p=0.73)$. However, contests involving a smaller male with recent losing experience were significantly less likely to escalate compared to those with a smaller naïve male $(G=16.71, \mathrm{df}=1, p<0.001)$. Smaller opponent size had a significant effect on an encounter escalating to a grapple, when the smaller opponent was a naïve fighter, with the probability of escalation increasing with the size of the smaller opponent. In contrast, the size of the smaller or larger opponent had no significant effect on contest escalation when the smaller opponent had previous fighting experience (Table 1, letter B).

\section{Outcome of grapples}

In contests that escalated to a grapple, those between two naïve males were won by the larger opponent in $72 \%$ of cases (binomial test: $p<0.001 ; n=107$ ). Similarly, contests involving a smaller, experienced opponent were won by the larger opponent in $71 \%$ of cases (binomial test: $p=0.01, n=44$ ). Recent fighting experience by the smaller opponent therefore had no influence on the outcome of grapples $(G=0.04, \mathrm{df}=1, p=0.852)$. In contests between naive males, the size of both the smaller and larger opponent significantly predicted contest outcome (Table 1, letter C). Smaller and larger opponent size had opposite effects of similar magnitude, so we performed a simple logistic regression using the size ratio to predict fight outcome $\left(\chi^{2}=10.621, \mathrm{df}=1, p=0.001\right.$, $b=9.34 \pm 2.87)$. In contrast, for contests involving an experienced male, neither smaller nor larger opponent size was a significant predictor of grapple outcome (Table 1, letter C).

\section{Overall larger male advantage}

Pooling both non-physical and physical encounters showed a clear pattern that the larger opponent wins more often, with $66 \%$ of contests involving two naïve males (binomial test: $p<0.001, n=149$ ) and $78 \%$ involving a smaller, experienced opponent (binomial test: $p<0.001, n=90$ ) being won by the larger opponent. There 
was a weak, non-significant effect of the type of staged contest $(G=3.57, \mathrm{df}=1, p=0.06)$.

\section{Duration of grapples}

Mean grapple duration was not significantly different between contests involving a smaller, experienced opponent (16.4 $\pm 2.5 \mathrm{~s}$ (range: $0.26-51.5 \mathrm{~s}), n=44)$ and those where both opponents were naïve fighters $(14.9 \pm 1.7 \mathrm{~s}$, (range: $0.3-104.8 \mathrm{~s}), n=107)\left(t_{1,148}=0.85, p=0.397\right)$. This also suggests that fatigue from past contests is unlikely to explain the reduced likelihood of fight escalation in contests where the smaller opponent had previous fighting experience. Simple regressions revealed that grapple duration was not significantly predicted by loser size, winner size or size difference for contests involving two naïve males. Grapple duration was, however, significantly negatively correlated to the size difference of opponents in contests involving a smaller, experienced opponent (Table 2).

The effect of winning/losing experience on contest outcome and duration

\section{Last fight experience}

There was no significant difference in size between males that won or lost their last contest (won: $612.85 \pm 103.73 \mathrm{mg}$, $n=22$; lost: $620.08 \pm 89.13 \mathrm{mg}, n=68, t_{1.8}=0.294, p=0.771$ ). Males with a win as their last fight experience were significantly more likely to win a non-physical encounter ( $3 / 7$ vs $4 / 39 ; G=3.881, \mathrm{df}=1, p=0.049)$ and were also more likely to escalate into a grapple $(15 / 22$ vs $29 / 68$; $G=4.41$, $\mathrm{df}=1, p=0.036)$ during the final staged contest. There was no effect, however, of last fight experience on winning final staged contests that escalated into grappling (6/15 vs $7 / 29$ $G=1.168, p=0.28$ ). Across all encounters, males that had won their last contest were more likely to win the final staged contest ( $9 / 22$ vs $11 / 68 ; G=5.39, \mathrm{df}=1, p=0.02)$.

\section{Overall fighting experience}

Male size had no effect on the proportion of contests won in the six previous encounters $\left(F_{1,83}=3.097, p=\right.$ 0.28). However, the proportion of previous contests won had a significant effect on contest outcome in nonphysical encounters during the final staged contest, with males with more wins being more likely to win nonphysical encounters in the staged fight $\left(\chi_{1}^{2}=7.00, p=0.008\right.$, $b=5.591 \pm 2.098)$. There was no effect of the proportions of wins on whether the final staged contest escalated into a grapple $\left(\chi_{1}^{2}=1.637, p=0.201, b=0.809 \pm 0.632\right)$, or on the outcome of contests that escalated into a grapple $\left(\chi_{1}^{2}=\right.$ 1.323, $p=0.25, b=1.008 \pm 0.876)$. Across all encounters, the males that won higher proportions of their previous contests were more likely to win during the final staged contest $\left(\chi_{1}^{2}=8.583, p=0.003, b=2.256 \pm 0.77\right)$.

\section{The effect of fighting experience on grapple duration}

An experienced male that had won his last contest fought for significantly longer in the final staged contest than a male that had lost his last fight $\left(t_{1,42}=2.973, p=0.005\right.$, recent winners: $26.4 \pm 4.4 \mathrm{~s}, n=15$; recent losers: $11.9 \pm 2.6 \mathrm{~s}$, $n=29)$. Similarly, the proportion of wins that a male had previously experienced was positively related to grapple duration in the final staged contest $\left(r^{2}=0.112, F_{1,39}=4.938\right.$, $p=0.032$ ).

Table 2 The effect of loser size, winner size and the size difference between opponents on grapple duration in contests involving either a smaller, naïve male $(n=107)$ and those when the smaller male has previous fighting experience $(n=44)$

\begin{tabular}{|c|c|c|c|c|c|c|}
\hline Predictor variables & $b(\mathrm{SE}) \times 1,000$ & $t$ value & $p$ & Effect size $(r)$ & $Z$ value & $p$ (effect size difference) \\
\hline \multicolumn{7}{|l|}{ Contest duration } \\
\hline \multicolumn{7}{|l|}{ Winner size } \\
\hline Naïve smaller opponent & $0.530(0.494)$ & 1.064 & 0.290 & 0.104 & \multirow[t]{2}{*}{1.621} & \multirow[t]{2}{*}{0.105} \\
\hline Experienced smaller opponent & $-1.329(1.075)$ & 1.236 & 0.223 & -0.192 & & \\
\hline \multicolumn{7}{|l|}{ Loser size } \\
\hline Naïve smaller opponent & $0.685(0.523)$ & 1.218 & 0.226 & 0.119 & \multirow[t]{2}{*}{0.790} & \multirow[t]{2}{*}{0.429} \\
\hline Experienced smaller opponent & $1.193(0.671)$ & 1.699 & 0.097 & 0.259 & & \\
\hline \multicolumn{7}{|l|}{ Winner size-loser size } \\
\hline Naïve smaller opponent & $-0.003(0.393)$ & 0.009 & 0.993 & -0.001 & \multirow[t]{2}{*}{1.837} & \multirow[t]{2}{*}{0.068} \\
\hline Experienced smaller opponent & $-1.211(0.560)$ & 2.165 & 0.036 & 0.324 & & \\
\hline
\end{tabular}

The $Z$ values and $p$ values in the last two columns are a test for a difference in effect size between the two types of staged contests. Significant $p$ values are in italics 


\section{Discussion}

In this study, we investigated whether recent fighting experience changed the role of body size in predicting contest behaviour in the field cricket T. commodus. During non-physical encounters, we found that smaller naïve males were more likely to win against a larger opponent and there was a higher probability of the contest escalating into a grapple, compared to encounters involving a smaller, experience male. Furthermore, in accordance with our predictions, experienced males with previous winning experience were more likely to win non-physical encounters, and these escalated into aggressive physical grapples more often than those where the male had previously lost. Once a contest escalated into an aggressive grapple, however, the effect of previous wins and losses was no longer apparent. Body size alone predicted contest outcome. It is possible that other factors, such as fatigue from previous contests, might have contributed to differences in contest behaviour and outcome. However, grapple duration did not differ between the two groups, strongly suggesting that fighting motivation as a result of previous fighting experience rather than exhaustion was the main factor contributing to the observed differences.

Body size is often considered the most reliable predictor of fighting ability in field crickets, including T. commodus (Shackleton et al. 2005), with larger opponents readily winning contests over smaller opponents (Hack 1997; Hofman and Schildberger 2001). We found, however, that a smaller, naïve opponent was as likely to win a nonphysical encounter as a larger, naïve opponent. Only when the smaller opponent had recent fighting experience did the larger opponent win the majority of contests that terminated prior to physical grappling. Thus, a larger male advantage during the early stages of assessment only occurred when the smaller opponent had recent fighting experience.

Smaller, naïve males may be more likely to win nonphysical encounters against a larger opponent if their motivation to fight is higher than a smaller, experienced male. At the start of a contest, males acquire information from physical contact of antennae. This signal is not energetically costly, so it is unlikely to be related to the actual fighting ability of a male but rather his motivation to fight (Hofman and Schildberger 2001). Furthermore, neither opponent's size predicted contest outcome in nonphysical encounters, irrespective of whether the smaller male was naïve or experienced. This suggests that body size is not assessed during these non-physical encounters. Both overall fighting experience and last fighting experience, however, influenced the outcome of non-physical encounters. Although the proportion of experienced males that were overall winners and losers was approximately equal (46\% vs $54 \%)$, the majority of the males $(76 \%)$ lost their last contest before the final staged contest. Male $T$. commodus that lost their last contest were more likely to lose a non-escalated encounter in the final staged contests compared to smaller naïve males or males that had experienced a recent win. Thus, the majority of experienced males were possibly less motivated to fight (because of recent losing experience) compared to smaller, naïve males that had not yet encountered an opponent. Similar results have been found in $R$. marmoratus, where recent fighting experience had a more significant effect on contest outcome than earlier fighting experience (Hsu and Wolf 1999). Recent fighting experience may have a stronger effect on the outcome of encounters than overall fighting experience as a result of a temporal decay in winner-loser effects.

Smaller, experienced males were also less likely to engage in an aggressive grapple with a larger opponent. The probability of contests escalating increased with the size of the smaller opponent when both males were naïve fighters. However, this effect disappeared when the smaller male had recent fighting experience, suggesting that experience is a major factor in determining contest escalation. More specifically, the type of experience had a significant effect on the probability of contests escalating. While overall fighting experience had no effect on contest escalation, males that lost their last contest were less likely to escalate to grappling in the final staged contest compared to smaller naïve males or smaller males that had won their previous encounter. Hsu and Wolf (2001) similarly found that male $R$. marmoratus that lost their last contest were less likely to escalate during the next contest compared to males that won their last contest. The smaller percentage of staged contests that escalate into a grapple when the smaller opponent had previous fighting experience is therefore probably attributable to the majority of experienced, smaller male $T$. commodus having lost their last fight and therefore being less motivated to escalate in the final staged encounter.

Once encounters escalated into a grapple, approximately $70 \%$ of the contests were won by the larger opponent, regardless of whether or not the smaller opponent had previous fighting experience. The exact nature of recent fighting experience (i.e. winner or loser) had no effect on contest outcome once an encounter escalated to grappling. Past experience therefore only significantly affected the early stages of a contest and the probability that it escalated. Fighting ability (RHP) based on body size appears to determine the eventual outcome of escalated encounters (grapples). In contrast to the apparent role of selfassessment in fight escalation, the outcome of grapples was predicted by the size ratio of the opponents when both males were naïve. This suggests that mutual assessment of opponent RHP was occurring. However, neither smaller nor larger opponent size predicted contest outcome when the smaller male had recent fighting experience. Analysis of 
grapple duration showed similar results when the smaller male had previous fighting experience, as only the size difference between opponents was significantly negatively correlated with fight duration, suggesting mutual assessment occurred during these contests.

It is important to note, however, that direct comparison of effect sizes revealed no significant difference in the role of body size on contest outcome or grapple duration between the two types of staged contests. Differences in RHP have been found to predict contest outcome and duration in other field cricket species (Hack 1997; Hofman and Schildberger 2001; Briffa 2008). It is therefore likely that mutual assessment occurs in T. commodus, as the majority of fights were won by the larger opponent in both types of staged contest and past experience had no effect on the outcome of these encounters. Nonetheless, differentiating between mutual and self-assessment using only RHP measures and contest duration remains inconclusive (Briffa and Elwood 2009). For example, the cumulative assessment model (CAM), which is based on self-assessment, also produces a negative relationship between winner and loser size, as found in mutual assessment. In this case, each opponent causes its rival to reach a 'giving up' threshold by inflicting costs, such as injuries. If the ability to do this varies with relative size, it will give a similar result to that for mutual assessment. Differentiating between mutual and self-assessment in future studies will therefore require more detailed information on contest structure and escalation and de-escalation within and between stages (Briffa and Elwood 2009).

Fighting evokes a suite of physiological changes in energetic resources, and differences between winners and losers in these measures appear to play an important role in the decision to retreat from a contest. In the field cricket, Acheta domesticus, for example, winners had higher glucose levels than losers, indicating that winners may have greater energy reserves (Briffa 2008). Lower energy reserves in losers might explain why experienced males lost non-physical encounters, as well as their reluctance to engage in escalated, aggressive encounters. Interestingly, we also found that previous winners fought for longer than previous losers during grapples, suggesting that these males might have higher energy levels than losers. However, differences in energy levels might be insufficient to overcome the effect of body size once a contest escalates, so that males can accurately assess their opponents' fighting abilities.

While previous studies on field crickets have shown that males which experienced a win in their last encounter were more likely to win a subsequent contest and vice versa (e.g. Savage et al. 2004), our study showed that recent fighting experience only affected contest outcome during non-physical encounters. Once a male could directly assess his opponent's fighting ability during a physical encounter, recent fighting experience did not alter the effect of body size on contest behaviour and outcome. Recent fighting experience therefore appears to affect perceived fighting ability in $T$. commodus. Fighting experience is an important factor affecting the role of body size in determining contest behaviour and outcome in many taxa (e.g. Schuett 1997; Hoefler 2002; Seebacher and Wilson 2007), and future studies should consider the relative influence of body size and fighting experience during each stage of a contest.

Acknowledgments This research was funded by the Australian Research Council (MDJ) and a Joyce Vickery Scientific Research Fund from the Linnaean Society of New South Wales (LTR). We thank James Davies for assistance in maintaining cricket stocks. We also thank Mark Briffa and two anonymous referees for valuable comments that greatly improved this manuscript.

\section{References}

Arnott G, Elwood RW (2009) Assessment of fighting ability in animal contests. Anim Behav 77:991-1004

Bakker Th CM, Bruijn E, Sevenster P (1989) Asymmetrical effects of prior winning and losing on dominance in sticklebacks (Gasterosteus aculeatus). Ethology 82:224-229

Briffa M (2008) Decisions during fights in the house cricket, Acheta domesticus: mutual or self assessment of energy, weapons and size? Anim Behav 75:1053-1062

Briffa M, Elwood RW (2001) Decision rules, energy metabolism and vigour of hermit-crab fights. Proc R Soc Lond B 268:1841-1848

Briffa M, Elwood RW (2009) Difficulties remain in distinguishing between mutual and self-assessment in animal contests. Anim Behav 77:759-762

Chase ID, Bartolomeo C, Dugatkin LA (1994) Aggression interactions and inter-contest interval: how long to winners keep winning? Anim Behav 48:393-400

Daws AG, Grills J, Konzen K, Moore PA (2002) Previous experience alters the outcome of aggressive interactions between males in the crayfish, Procambarus clarkii. Mar Fresh Behav Physiol 35:139-148

Elias DO, Kasumovic MM, Punzalan D, Andrade MCB, Mason AC (2008) Assessment during aggressive contests between male jumping spider. Anim Behav 76:901-910

Hack MC (1997) Assessment strategies in the contests of male crickets, Acheta domesticus (L). Anim Behav 53:733-747

Hoefler CD (2002) Is contest experience a trump card? The interaction of residency status, experience, and body size on fighting success in Misumenoides formosipes (Araneae: Thomisidae). J Insect Behav 15:779-790

Hofman HA, Schildberger K (2001) Assessment of strength and willingness to fight during aggressive encounters in crickets. Anim Behav 62:337-348

Hofman HA, Stevenson PA (2000) Flight restores fight in crickets. Nature 403:613

Hsu Y, Wolf LL (1999) The winner and loser effect: integrating multiple experiences. Anim Behav 57:903-910

Hsu Y, Wolf LL (2001) The winner and loser effect: what fighting behaviours are influenced? Anim Behav 61:777-786 
Hsu Y, Early RL, Wolf LL (2006) Modulation of aggressive behaviour by fighting experience: mechanisms and contest outcomes. Biol Rev 81:33-74

Hsu Y, Lee IH, Lu CK (2009) Prior contest information: mechanisms underlying winner and loser effects. Behav Ecol Sociobiol 63:1247-1257

Humphries EL, Hebblethwaite AJ, Batchelor TP, Hardy ICW (2006) The importance of valuing resources: host weight and contender age as determinants of parasitoid wasp contest outcomes. Anim Behav 72:891-898

Kasumovic MM, Elias DO, Punzalan D, Mason AC, Andrade MCB (2009) Experience affects the outcome of agnostic contests without affect the selective advantage of size. Anim Behav 77:1533-1538

Pomfet J, Knell RJ (2006) Sexual selection and horn allometry in the dung beetle Euoniticellus intermedius. Anim Behav 71:567-576

Prenter J, Elwood RW, Taylor PW (2006) Self-assessment by males during energetically costly contest over precopula females in amphipods. Anim Behav 72:861-868

Rutte C, Taborsky M, Brinkhof MWG (2006) What sets the odds of winning and losing? Trends Ecol Evol 21:16-21
Savage KE, Hunt J, Jennions MD, Brooks R (2004) Male attractiveness covaries with fighting ability but not prior fight outcome in house crickets. Behav Ecol 16:196-200

Schuett GW (1997) Body size and agonistic experience affect dominance and mating success in male copperheads. Anim Behav 54:213-224

Seebacher F, Wilson RS (2007) Individual recognition in crayfish (Cherax dispar): roles of strength and experience in deciding aggressive encounters. Biol Lett 3:471-474

Shackleton MA, Jennions MD, Hunt J (2005) Fighting success and attractiveness as predictors of male mating success in the black field cricket Teleogryllus commodus: the effectiveness of nochoice tests. Behav Ecol Sociobiol 58:1-8

Taylor PW, Elwood RW (2003) The mismeasure of animal contests. Anim Behav 65:1195-1202

Whitehouse MEA (1997) Experience influences male-male contest in the spider Argyrodes antipodiana (Theridiidae: Araneae). Anim Behav 53:913-923

Zar JH (1999) Biostatistical analysis, 4th edn. Prentice Hall, Upper Saddle River 